\title{
Reflujo vesicoureteral esencial y reimplantación ureteral extravesical en niños
}

\author{
García Mérida $\mathrm{M}^{*}$, Gosalbez Jr. R**, Rius Díaz F***, Labbie A**, Castellán $\mathrm{M}^{* *}$. \\ *Sección de Urología Pediátrica. Hospital Universitario Materno-Infantil de Málaga. **División de Urología \\ Pediátrica. Miami Children's Hospital y Jackson Memorial Hospital, Universidad de Miami, Florida, USA. \\ ${ }^{* * *}$ Cátedra de Bioestadística. Departamento de Medicina Preventiva y Salud Pública. Universidad de Málaga.
}

Actas Urol Esp 2006; 30 (6): 602-609

\begin{abstract}
RESUMEN
REFLUJO VESICOURETERAL ESENCIAL Y REIMPLANTACIÓN URETERAL EXTRAVESICAL EN NIÑOS

Objetivos: Conocer los resultados de la reimplantación extravesical de Lich-Gregoire en el tratamiento quirúrgico del reflujo vesicoureteral (RVU) primario.

Material y Métodos: En un período de 2,5 años se realizó un estudio retrospectivo no randomizado de 141 niños con RVU esencial sometidos a reimplante ureteral: 101 niños (158 uréteres refluyentes) por la técnica de Lich-Gregoire y 40 (68 uréteres) por la de Cohen. Se analizan los datos de los reimplantes extravesicales y se valoran: edad, indicaciones cirugía, días con sonda vesical, estancia hospitalaria y complicaciones a corto y largo plazo.

Resultados: El tiempo medio de control ha sido de 1,71 años (Rango 8 meses a 3,5 años).

A) Complicaciones precoces. No hubo ningún caso de obstrucción ureterovesical. Cinco niños $(8,6 \%$ de los operados bilateralmente de forma simultánea) presentaron retención urinaria pero sólo tres necesitaron recolocación de sonda vesical y uno sólo de sondaje intermitente temporal. Las nauseas y vómitos, el dolor y la hematuria fueron escasos.

B) Complicaciones tardías. Los resultados a largo plazo son buenos en el 95\%. Se observó RVU persistente en 7 uréteres $(4,4 \%)$ y RVU contralateral en 3 niños $(6,7 \%$ de los casos unilaterales). Sólo precisaron nuevo tratamiento quirúrgico 3 uréteres $(2 \%)$ con RVU ipsilateral persistente.

Se compararon los resultados de la técnica de Lich-Gregoire con la de Cohen y se observó que en la extravesical eran significativamente menores el número de complicaciones, dolor, hematuria, días con sonda vesical y estancia hospitalaria.

Conclusiones: La técnica de Lich-Gregoire es un procedimiento de corta estancia hospitalaria, efectivo para la corrección del RVU, que origina menos molestias, dolor, hematuria y días de ingreso que las técnicas intravesicales. No obstante puede ocasionar disfunción vesical transitoria en los casos bilaterales.

Palabras clave: Reflujo vesicoureteral. Reimplantación ureteral. Disfunción miccional. Retención aguda urinaria. Uréter, vejiga. Técnica quirúrgica.
\end{abstract}

\section{ABSTRACT}

PRIMARY VESICOURETERAL REFLUX AND EXTRAVESICAL URETERAL REIMPLANTATION IN CHIDREN

Purpose: to assess the results of the Lich-Gregoire procedure in the treatment of primary vesicoureteral reflux (VUR).

Materials and methods: In a 2.5-year period, 141 children with primary VUR underwent a vesicoureteral reimplantation using the Lich-Gregoire procedure in 101 patients (158 ureters) and the Cohen procedure in 48 patients (68 ureters). Patients were evaluated retrospectively in a non randomized fashion and data were recorded about: age, indications for surgery, days with the bladder catheter, length of stay, and short and long-term complications.

Results: The control average time was 1.71 years (from 8 months to 3.5 years).

A) Early complications. No obstruction was seen in this series. Five children (8.6 of the bilaterally simultaneously operated) showed urinary retention, but only three needed replacement of the bladder catheter and only one of them needed temporary clean intermittent catheterization. Nausea, vomiting, pain and hematuria were sporadic and limited in time.

B) Late complications. The long-term results were good (95\%). Seven ureters (4.4\%) had persistent VUR and 3 children $(6.7 \%$ of the unilateral cases) had contralateral VUR. Only 3 ureters needed a new surgical treatment (2\%) for persistent ipsilateral VUR.

Short and long-term complications, days with bladder catheter and length of stay in the hospital were significantly smaller in the group of patients operated with Lich-Gregoire procedure than in patients operated with the Cohen technique.

Conclusions: The Lich-Gregoire procedure is a technique associated with shorter postoperative hospitalization and less discomfort, pain and hematuria than the intravesical technique. Both techniques were effective in correcting VUR. Extravesical reimplantation can cause transitory bladder dysfunction in a small percentage of the bilateral cases.

Keywords: Vesicoureteral Reflux. Ureteral Reimplantation. Voiding Dysfunction. Urinary Retention. Ureter.

Cuadro de abreviaturas: Bil: Bilateral. Contralat: Contralateral. ITU: Infección tracto urinario. №: Número. NS: no significativo. PO: Postoperatorio. RVU: Reflujo vesicoureteral. UUV: Unión uretererovesical. 
$\mathrm{E}$ xisten varias técnicas quirúrgicas abiertas para la corrección del RVU, pero las más utilizadas en niños son los procedimientos intravesical de Cohen, extravesical de Lich-Gregorire y el mixto de Politano-Ledbetter ${ }^{1,2}$. La reimplantación extravesical descrita por Lich-Gregoire ${ }^{3,4}$ o sus variantes ${ }^{5}$ tienen ventajas sobre las intravesicales y por ello está ganando adeptos. En ésta no se abre la vejiga y el riesgo de contaminación de la herida es menor, el tiempo quirúrgico más corto, el postoperatorio mejor tolerado (menos hematuria, menos espasmos del detrusor, menores molestias), precisa menor mediación analgésica postoperatoria y, finalmente, requiere menos días de sondaje y hospitalización ${ }^{3}$. No obstante, también tiene inconvenientes como son la retención urinaria postoperatoria transitoria en los casos de reimplantación bilateral simultánea y no poder observar la vejiga para valorar enfermedades asociadas como divertículos, meatos contralaterales anómalos, etc. ${ }^{6,7}$; por ello es necesario, en algunos casos, realizar cistouretroscopia previa, en el mismo acto quirúrgico.

\section{OBJETIVOS}

Se realizó un estudio retrospectivo no randomizado de los pacientes sometidos a reimplante ureteral con objeto de conocer los resultados a corto y largo plazo, tiempo de sondaje vesical y días de estancia hospitalaria.

\section{MATERIAL Y MÉTODOS}

En un periodo de dos años y medio (junio 1998 a enero 2001) se intervinieron 171 niños con RVU de distinta etiología en la División de Urología Pediátrica del Miami Children's Hospital. De ellos, 141 presentaron RVU esencial y se sometieron a reimplantación ureteral: 101 por la técnica de Lich-Gregoire y 40 por la de Cohen. Se analizan minuciosamente los 101 pacientes que fueron tratados con el procedimiento extravesical y se comparan sus resultados con el intravesical. Se valoran edad, grado de RVU, indicaciones de cirugía, procedimientos asociados, días de sondaje vesical, estancia hospitalaria y resultados a corto y largo plazo. Se realizó estudio estadístico empleando el Chi-cuadrado de Pearson para las variables cualitativas y la $\mathrm{T}$ de Student para las cuantitativas; para las variables de respuesta múltiple se utilizó un contraste de dos proporciones para cada uno de los ítems de posibles respuestas.

Los pacientes fueron estudiados preoperatoriamente con ultrasonidos, cistograma y gammagrafía isotópica con ácido dimetilmercaptosuccinico (DMSA).

El antirreflujo extravesical se realizó por la técnica de Lich-Gregoire en Y invertida sin desinserción del uréter. Aunque la técnica es la clásica descrita por los autores ${ }^{3,4}$, el procedimiento sigue los siguientes pasos: colocación de sonda de Foley intraoperatoria para controlar la distensión vesical. A través de una incisión de Pfannestiel se llega hasta la vejiga y se diseca la cara anterior y el lateral correspondiente. Se vacía vejiga. Se localizan los vasos iliacos externos reflejando el peritoneo cranealmente para localizar el uréter y se coloca el separador de Dennis-Brown. Se identifica la arteria umbilical obliterada, que de ser necesario se liga y secciona. El uréter se localiza fácilmente debajo de la arteria umbilical, entre la vejiga y el peritoneo. Se tracciona el uréter con un vessel loop y se diseca ventralmente hasta su inserción y en una longitud de 3 a 4 centímetros, evitando abusar del bisturí eléctrico. En casos bilaterales, sólo se usa cauterización con pinzas bipolares. Cerca de la unión uretero-vesical (UUV) existen unos vasos, provenientes de la arteria vesical inferior, que se deben ligar o cauterizar para evitar hematomas indeseables. Como la vejiga está retraída hacia el lado contrario al que se va a realizar el reimplante hay que diseñar el sentido del túnel para evitar angulaciones y obstrucciones ureterales posteriores al colocar la vejiga en su posición normal. Apertura de la capa superficial del detrusor con bisturí eléctrico de aguja fina y continuar la separación muscular hasta la mucosa con tijeras y ángulo recto; con la vejiga medio llena la mucosa protuye hacia fuera y es más fácil la disección. Hay que disecar bien la cara ventral y un poco los laterales de la inserción ureteral como en forma de Y invertida; no se debe dejar ninguna fibra de detrusor en la UUV para que el uréter entre bien en la vejiga al aproximar el detrusor y para que sus fibras no ocasionen obstrucción ureteral al cerrar el neotunel. Sí hay que efectuar modelaje se desinsertará el uréter de la vejiga. La longitud del túnel debe tener una 
proporción de 4-5 a 1 en relación al diámetro del uréter. Si se abre la mucosa vesical no se debe suturar, pues el agujero se hace mayor; se colocará un mosquito y se cerrará con una ligadura de material reabsorvible de 5-0. Cierre del detrusor con 4 a 6 puntos sueltos de vicryl o monocryl 4/0 y comprobar con un ángulo recto que no existe obstrucción ureteral proximal. Se coloca la vejiga en posición normal y se comprueba que el uréter no entra acodado en la vejiga. Se realiza hemostasia cuidadosa y se procede al cierre por planos sin colocar ningún tipo de drenaje. Se deja sonda vesical de Foley de entre 24-48 horas en los casos unilaterales y 48-72 en los bilaterales.

En casi todos los pacientes sometidos a reimplantación ureteral extravesical se realizó cistouretroscopia previa para conocer la posición y aspecto de los meatos ureterales, la longitud del túnel submucoso y, además para conocer la existencia de otras malformaciones asociadas (divertículos, litiasis, duplicidades, etc.).

$\mathrm{El}$ antirreflujo intravesical se realizó siempre por la técnica de Cohen; la sonda vesical, tipo Foley, se dejó tres días en los unilaterales y cuatro en los bilaterales. En casos selectivos (modelaje) se dejaron tutores ureterales externos (sonda de Nelaton).

En el postoperatorio inmediato todos los pacientes fueron sometidos a tratamiento con cloruro de oxibutinina mientras tenían sonda vesical o tutor ureteral, antibióticos y analgésicos (paracetamol con codeína). Nunca se empleó el bloqueo caudal ni la infiltración de la herida con anestesia tópica. En los casos bilaterales y de riñón único se determinó la creatinina plasmática. Postoperatoriamente se prescribió tratamiento con antibióticos a dosis profilácticas hasta que se demostró la curación del RVU en el cistograma postoperatorio. Todos los pacientes se controlaron con ultrasonidos a los tres meses y cistografía isotópica al sexto mes.

\section{RESULTADOS}

Los 101 pacientes sometidos a reimplantación extravesical fueron $62(61,4 \%)$ del sexo femenino y $39(38,6 \%)$ del masculino; el lado fue derecho en $17(16,8 \%)$, izquierdo en $27(26,7 \%)$ y bilateral en $57(56,5 \%)$ reuniendo un total de 158 unidades renales refluyentes. Hay tres casos de riñón único. El grado de RVU se especifica en la Tabla 1.
Tabla 1

\begin{tabular}{cc}
\hline Grados de reflujo & n (porcentaje) \\
\hline I. & $6(3,8 \%)$ \\
II. & $53(33,5 \%)$ \\
III. & $57(36,1 \%)$ \\
IV & $29(18,4 \%)$ \\
V & $13(8,2 \%)$ \\
\hline
\end{tabular}

Los ultrasonidos mostraron nefropatía en 45 niños con un total de 49 riñones afectos (31\%). El DMSA se realizó en 92 niños y la función renal diferencial media fue de 55,5\% (Rango 12 a 85\%) para el lado derecho y de 48,5\% (Rango 15-88\%) para el izquierdo; existía nefropatía en 57 niños con un total de 69 (44\%) riñones afectos. La nefropatía fue más frecuente en los altos grados de RVU y en los niños más pequeños.

Las indicaciones de cirugía fueron varias para cada paciente y están reflejadas en la Tabla 2 . Ningún paciente tenía historia de disfunción miccional previa. Todos los RVU grado I operados tenían un RVU contralateral de más alto grado.

La edad media de la cirugía fue de 5,4 años (Rango 4 meses a 24 años). En 75 niños se realizó cistouretroscopia previa, que no evidenció anomalías importantes, y en 5 modelaje ureteral. Se dejó doble J, con hilo externo, durante una semana, en los uréteres modelados, en los riñones únicos y en un RVU bilateral de alto grado; el catéter se retiraba a los 7 días en la policlínica.

Setenta y nueve niños tuvieron un postoperatorio inmediato sin incidencias; 22 niños $(22 \%)$ presentaron 28 complicaciones inmediatas (Tabla 3). Un paciente, sometido a reimplantación bilateral,

Tabla 2

\begin{tabular}{lc}
\hline Indicaciones de cirugia & n (porcentaje) \\
\hline Nefropatía & $49(49 \%)$ \\
Grado de RVU & $42(42 \%)$ \\
UTI recidivantes & $39(39 \%)$ \\
Edad de presentación y duración del RVU & $25(25 \%)$ \\
Los padres no quieren continuar & \\
con tratamiento médico & $11(11 \%)$ \\
No respuesta al tratamiento antibiótico & $11(11 \%)$ \\
Otras & $10(10 \%)$ \\
$\quad$ Pubertad & 5 \\
$\quad$ Riñón único & 3 \\
$\quad$ Hipertensión arterial & 1 \\
$\quad$ Plastia pieloureteral previa & 1 \\
\hline
\end{tabular}


Tabla 3

Complicaciones precoces reimplantación extravesical

\begin{tabular}{lc}
\hline & n (porcentaje) \\
No & $79(78,2 \%)$ \\
Si & $22(21,8 \%)$ niños / \\
& 28 complicaciones \\
Náuseas/vómitos & $11(11 \%)$ \\
Dolor (espasmo vesical) & $10(10 \%)$ \\
Retención urinaria & $5(5 \%)$ \\
Hematuria & $1(1 \%)$ \\
Obstrucción transitoria & $1(1 \%)$ \\
\hline
\end{tabular}

presentó oligo-anuria transitoria durante 22 horas que se solucionó espontáneamente. Cinco reimplantaciones bilaterales presentaron retención urinaria al retirar la sonda de Foley a las 72 horas de la intervención (Tabla 4). De estos pacientes uno comenzó a orinar a las 24 horas, otro a las 48 y los tres restantes necesitaron recolocación de sonda vesical por ascenso de las cifras de creatinina plasmática; dos se curaron al séptimo día y el restante al noveno, pero precisó sondaje vesical intermitente durante 4 semanas hasta su curación, y posteriormente presentó un hábito miccional normal.

La sonda de Foley se dejó una media de 2,6 días (Rango 2-9 días) y la estancia hospitalaria media fue de 2,86 días (Rango 2-9 días). Los resultados de los US practicados al tercer mes de postoperatorio se expresan en la Tabla 5.

Un paciente se perdió de control y 10 uréteres (6,9\%) presentaron complicaciones tardias: 7 $(4,4 \%)$ tuvieron RVU persistente y 3 (6,8\% de unilaterales) RVU contralateral; todos fueron de grado I a III. Procedimientos debidos a las complicaciones sólo precisaron tres niños con RVU ipsilateral persistente y en todos se realizó antirreflujo endoscópico con dextranómero del ácido hialurónico utilizando la técnica convencional.
Tabla 5

Ultrasonidos postoperatorios (3er mes)

\begin{tabular}{lc}
\hline & n (porcentaje) \\
Normal & $71(70 \%)$ \\
Igual que preoperatoriamente & $24(24 \%)$ \\
Aumento nefropatía & $4(4 \%)$ \\
Disminución dilatación & $2(2 \%)$ \\
\hline
\end{tabular}

Los RVU contralaterales curaron sin cirugía. En todos los pacientes reoperados remitió el RVU. El tiempo medio de control ha sido de 1,71 años (Rango 8 meses a 3,5 años).

En la Tabla 6 se exponen los resultados globales de toda la serie de reimplantaciones ureterales (extra e intravesicales) observando buenos resultados en el 95\% de los casos; en la Tabla 7 se especifican los resultados del reimplante extravesical aislado y existen unos resultados similares. El porcentaje de RVU postoperatorio persistente ipsilateral es también casi similar en ambos grupos de pacientes, oscilando entre el $4,4 \%$ y el 4,8\%. El porcentaje de RVU contralateral es más alto en los extravesicales $(6,8 \%)$ que en el total de la serie $(5,4 \%)$ aunque la diferencia no es estadísticamente significativa. Los niños con RVU persistente se sometieron a observación y sólo precisaron cirugía cinco niños (2\%), dos de ellos intravesicales, con reflujo ipsilateral. En todos se realizó antirreflujo endoscópico con dextranómero del ácido hialurónico con buen resultado final.

Se compararon los resultados de las reimplantaciones extravesicales (101 niños con 158 uréteres refluyentes) con las intravesicales (40 niños con 68 unidades renales refluyentes) operadas en la misma unidad:

Tabla 4

Casos que desarrollaron retención urinaria

\begin{tabular}{cccccccc}
\hline $\begin{array}{c}\text { Edad } \\
\text { en años }\end{array}$ & Sexo & $\begin{array}{c}\text { Grado } \\
\text { RVU }\end{array}$ & $\begin{array}{c}\text { Lado } \\
\text { Reimplante }\end{array}$ & $\begin{array}{c}\text { Tiempo } \\
\text { en dias }\end{array}$ & $\begin{array}{c}\text { Creatinina } \\
\text { sérica }\end{array}$ & Tto & Resultado \\
\hline 7 & Fem. & III Bil & Bilateral & 1 & $0,5 \mathrm{mg} / \mathrm{dl}$ & No & Curado \\
4 & Masc. & III Bil & Bilateral & 2 & $0,6 \mathrm{mg} / \mathrm{dl}$ & No & Curado \\
7 & Fem. & III/IV & Bilateral & 3 & $2,1 \mathrm{mg} / \mathrm{dl}$ & S. Foley & Curado \\
1 & Masc. & III/V & Bilateral & 3 & $3 \mathrm{mg} / \mathrm{dl}$ & S. Foley & Curado \\
5 & Fem. & IV/III & Bilateral & 5 & $6,7 \mathrm{mg} / \mathrm{dl}$ & S. Foley & Curado \\
\hline
\end{tabular}


Tabla 6

Reflujo vesicoureteral primario. Resultados globales de las reimplantaciones ureterales (extra e intravesicales)

\begin{tabular}{|c|c|c|c|c|c|c|c|c|c|c|c|}
\hline \multirow[t]{2}{*}{ Grado } & \multirow[t]{2}{*}{ Uréteres } & \multicolumn{2}{|c|}{ Eficacia } & \multirow{2}{*}{\multicolumn{2}{|c|}{$\begin{array}{cr}\text { RVU } & \text { PO } \\
\text { Ipsilateral }\end{array}$}} & \multirow{2}{*}{$\begin{array}{l}\text { RVU } \\
\text { Contra }\end{array}$} & \multirow{2}{*}{$\begin{array}{l}\text { PO } \\
\text { lateral }\end{array}$} & \multicolumn{2}{|c|}{ Observación } & \multicolumn{2}{|c|}{ Reoperación } \\
\hline & & No & $\%$ & & & & & № & $\%$ & No & $\%$ \\
\hline I & 6 & 6 & 100 & & & & & & & & \\
\hline II & 70 & 66 & 94,3 & 4 & $5,7 \%$ & 2 & $2,8 \%$ & 3 & 4,3 & 1 & 1,4 \\
\hline III & 78 & 73 & 93,6 & 5 & $6,4 \%$ & 1 & $1,3 \%$ & 3 & 3,8 & 2 & 2,6 \\
\hline IV & 48 & 47 & 97,9 & 1 & $2,0 \%$ & & & 1 & 2,0 & & \\
\hline $\mathrm{V}$ & 24 & 23 & 95,8 & 1 & $4,1 \%$ & & & 1 & 4,2 & & \\
\hline Total & 226 & 215 & $95,1 \%$ & 11 & $4,8 \%$ & 3 & $\mathbf{5 , 4} \%$ & 6 & $2,6 \%$ & 5 & $2,2 \%$ \\
\hline
\end{tabular}

Abreviaturas: №: número de casos; \% porcentaje; RVU: Reflujo Vesicoureteral; PO: postoperatorio; Contralat: contralateral.

Tabla 7

Reflujo vesicoureteral primario. Resultados reimplantaciones ureterales extravesicales

\begin{tabular}{|c|c|c|c|c|c|c|c|c|c|c|c|}
\hline \multirow[t]{2}{*}{ Grado } & \multirow[t]{2}{*}{ Uréteres } & \multicolumn{2}{|c|}{ Eficacia } & \multirow{2}{*}{\multicolumn{2}{|c|}{$\begin{array}{cr}\text { RVU } & \text { PO } \\
\text { Ipsilateral }\end{array}$}} & \multirow{2}{*}{$\begin{array}{l}\text { RVU } \\
\text { Contral }\end{array}$} & \multirow{2}{*}{$\begin{array}{l}\text { PO } \\
\text { lateral }\end{array}$} & \multicolumn{2}{|c|}{ Observación } & \multicolumn{2}{|c|}{ Reoperación } \\
\hline & & No & $\%$ & & & & & No & $\%$ & No & $\%$ \\
\hline I & 6 & 6 & 100 & & & & & & & & \\
\hline II & 53 & 51 & 96,2 & 2 & $5,7 \%$ & 2 & $3,8 \%$ & 2 & 4,3 & & \\
\hline III & 57 & 53 & 92,9 & 4 & $6,4 \%$ & 1 & $1,7 \%$ & 2 & 3,8 & 2 & 3,8 \\
\hline IV & 29 & 28 & 96,6 & 1 & $2,0 \%$ & & & & & & \\
\hline $\mathrm{V}$ & 13 & 13 & 100 & & & & & & & & \\
\hline Total & 158 & 151 & $95,6 \%$ & 7 & $4,4 \%$ & 3 & $6,8 \%$ & 4 & $\mathbf{2 , 5} \%$ & 3 & $1,9 \%$ \\
\hline
\end{tabular}

Abreviaturas: №: número de casos; \% porcentaje; RVU: Reflujo Vesicoureteral; PO: postoperatorio; Contralat: contralateral.

1. Complicaciones inmediatas (Tabla 8). Tuvieron complicaciones precoces 22 niños $(21,78 \%)$ de los extravesicales frente a $23(57,5 \%)$ de los intravesicales, diferencia que es estadísticamente significativa (p: <0,0001). El dolor y la hematuria fueron significativamente más frecuentes en los intravesicales que en los extravesicales. En náuseas y vómitos, retención urinaria y obstrucción no existieron diferencias significativas en ninguno de los dos grupos.

2. Comparación de días con sonda de Foley: La media fue de 2,6 días ( $R$ 2-9 días) en extravesicales y de 3,4 (R 2-7 días) en los intravesicales, diferencia estadísticamente significativa (p: <0,0001).

3. Comparación de días de ingreso hospitalario: La media en los extravesicales fue de 2,86 días (R 2-9) y en los intravesicales de 3,65 días (R 2-7), diferencia significativa (p: $<0,0001)$.
4. En las complicaciones tardías, RVU persistente ipsi o contralateral, no existen diferencias significativas en ninguno de los dos grupos.

\section{DISCUSIÓN}

Las metas actuales de los cuidados médicos son limitar la estancia hospitalaria y el coste sin comprometer la calidad de vida. Cuando se consideran las distintas técnicas de reimplantación ureteral abierta, el abordaje extravesical es menos mórbido que el intravesical por las razones enumeradas previamente ${ }^{3,7}$.
Tabla 8

Complicaciones inmediatas de los reimplantes. Comparación enre los extra e intravesicales

\begin{tabular}{|c|c|c|c|}
\hline & $\begin{array}{c}\text { Extravesicales } \\
101 \text { niños/ } \\
158 \text { uréteres }\end{array}$ & $\begin{array}{c}\text { Intravesicales } \\
40 \text { niños/ } \\
68 \text { uréteres }\end{array}$ & $\begin{array}{l}\text { Significación } \\
\text { estadistica }\end{array}$ \\
\hline № de niños & $\begin{array}{c}22(21,8 \%) \\
28 \text { complicaciones }\end{array}$ & $\begin{aligned} & 23 \quad(57,5 \%) \\
27 & \text { complicaciones }\end{aligned}$ & $\mathrm{p}<0,0001$ \\
\hline Náuseas y vómitos & 11 (11\%) & $3 \quad(7,5 \%)$ & NS \\
\hline Dolor & $10 \quad(10 \%)$ & $(27,5 \%)$ & $\mathrm{p}<0,04$ \\
\hline Hematuria & $(1 \%)$ & $(27,5 \%)$ & $\mathrm{p}<0,0001$ \\
\hline Retención urinaria & $5 \quad(5 \%)$ & $(2,5 \%)$ & NS \\
\hline Obstrucción & $1 \quad(1 \%)$ & $(2,5 \%)$ & NS \\
\hline
\end{tabular}

Abreviaturas: NS: no significativo 
Los principios fundamentales en cualquier cirugía antirreflujo son crear un túnel submucoso de longitud suficiente, que exista un soporte muscular firme del uréter y que éste quede sin tensión ${ }^{8-10}$. La longitud del túnel debe tener una proporción de 4 ó 5 en relación al diámetro del uréter $^{11}$. En esta serie se ha utilizado la técnica clásica original de Lich-Gregoire ${ }^{3,4}$, sin desinserción del uréter y con las puntualizaciones descritas en el material y métodos.

Las técnicas quirúrgicas abiertas para la corrección del RVU tienen un éxito del 95\% para los grados I a IV y del $80 \%$ para los grados V que precisan modelaje ${ }^{12}$. En los procedimientos extravesicales el porcentaje oscila entre el 90 y 99\% $\%^{7-9,13,14}$. En esta serie, el porcentaje de éxito del total de reimplantaciones y de los extravesicales solos son prácticamente similares, el 95\% para todos los grados de RVU, oscilando del $93 \%$ en el grado III al $100 \%$ en los grados I y V (Tablas 6 y 7).

El riesgo de obstrucción ureteral es prácticamente nulo en los procedimientos extravesicales y solo se han descrito ureterohidronefrosis transitorias en un $3 \%^{13}$; mientras que en los procedimientos intravesicales ocurre entre el 1 y $9 \%{ }^{15}$. Paradójicamente, las técnicas extravesicales son las más idóneas para colocar fácilmente un doble $\mathrm{J}$ en casos de obstrucción ureterovesical puesto que no se altera el trayecto longitudinal del uréter.

El RVU contralateral se presenta entre el 5,3 y $32 \%$ de todos los procedimientos antirreflujo ${ }^{6,15-17}$ y su etiología es desconocida; puede ocurrir por una distorsión quirúrgica del trígono durante el reimplante, por la existencia de un RVU contralateral previo desconocido, por la aparición de una disfunción vesical o porque al eliminar un RVU de alto grado aumenta la presión vesical y por el mecanismo de pop-off se desestabiliza en el uréter contralateral ${ }^{12,16-18}$. La incidencia varía según la técnica quirúrgica de reimplantación unilateral empleada: 0 a $19 \%$ en el Cohen, 9 a $27 \%$ en el LeadbetterPolitano, $17 \%$ en el Glen-Anderson, $20 \%$ en el Paquin, y $7 \%$ en el endoscópico ${ }^{15-18}$. Minevich et al. ${ }^{16}$, en una revisión de la literatura, encontraron una incidencia de RVU contralateral del $5,3 \%$ en 206 reimplantes extravesicales. Los resultados de esta serie son del 6,8\% de los reimplantes unilaterales (47 niños) y el lado operado tenía un RVU grado II en dos casos y grado III en el restante, por lo que se excluye el mecanismo del pop-off. Lógicamente en la literatura, el RVU contralateral es menos frecuente en los procedimientos que desestructuran menos el trígono como son los extravesicales y los endoscópicos ${ }^{12,16,17}$, pero en esta serie no se observó ningún caso en los reimplantes unilaterales realizados por la técnica de Cohen aunque la diferencia no es estadísticamente significativa. Para evitar esta complicación, sí existe un RVU contralateral previo, sea del grado que sea, se recomienda realizar reimplantación bilateral. La edad, el grado de RVU contralateral y el aspecto del meato ureteral no parecen jugar ningún papel en la génesis del mismo ${ }^{15-18}$. El RVU contralateral se resuelve espontáneamente en dos tercios de los casos y el tercio restante precisa cirugía ${ }^{12,15,16}$. En nuestra serie todos los RVU contralaterales se curaron con actitud expectante y tratamiento antibiótico profiláctico.

La retención urinaria es la complicación inmediata más temida de los procedimientos antirreflujo extravesicales, aunque es poco frecuente y transitoria, durando alrededor de un mes como máximo; precisan sondaje intermitente un 26\% de las mismas $5,13,14,19$. Es la complicación más grave pues puede ocasionar ureterohidronefrosis bilateral e insuficiencia renal aguda sí no se realiza el sondaje vesical, como ocurrió en tres pacientes de esta serie. No se ha descrito en los procedimientos extravesicales unilaterales ni en la técnica de Cohen uni o bilateral ${ }^{8,14,18-21}$; sólo se presenta en los reimplantes extravesicales bilaterales realizados de forma simultánea ${ }^{5,10,14,19}$ con una prevalencia del 1,3 al $26 \% \%^{5-10,13,14,22}$. Nuestra incidencia es del 8,6\% de los reimplantes bilaterales extravesicales, pero sólo 3 niños $(5,2 \%)$ necesitaron recolocación de sonda de Foley y uno solo $(1,7 \%)$ precisó posteriormente de sondaje limpio intermitente durante un mes. Todos los pacientes recuperaron su patrón miccional normal. También se ha descrito disfunción vesical en el $2,1 \%$ de los pacientes sometidos al Politano, probablemente por ser una técnica combinada, intra y extravesical ${ }^{19,20}$. 
Se han realizado estudios en las distintas variantes del Lich-Gregoire y se ha observado que la retención urinaria es algo más frecuente en la variante de desinserción y avance ureteral de Zaont, pues en ésta la disección de la UUV es mayor, pero las diferencias no son estadísticamente significativas $6,13,19$.

Se ha demostrado que es más frecuente en RVU de alto grado, niños menores de 3 años y en el sexo masculino ${ }^{13}$ pero estos datos no coinciden con esta serie pues sólo hay un niño menor de 3 años, el $60 \%$ son de alto grado en un solo lado y otro 60\% son niñas (Tabla 4). La retención urinaria postoperatoria no influye en la resolución del $\mathrm{RVU}^{8}$.

Se desconoce el mecanismo de la disfunción vesical pero se cree que es debida a una alteración de la inervación vesical ${ }^{9,10,19-21}$. Se han realizado varios estudios anatómicos y experimentales para conocer la neuroanatomía y neurofisiología de esta zona y poder encontrar una explicación fisiopatológica a la disfunción vesical ${ }^{19-21}$. Los estudios de Leissner et al. ${ }^{19}$, efectuados en cadáveres humanos, mostraron que el plexo pélvico, el responsable del vaciado vesical, está situado en la cara dorsal y medial de la UUV y ramas del mismo forman una red alrededor del uréter dorsal, trígono y recto. Yucel y Baskin ${ }^{20}$ en fetos humanos, de 21 a 40 semanas y con técnicas inmunohistoquímicas, encuentran una inervación muy similar. Los estudios experimentales de Martínez-Portillo ${ }^{21}$ comprobaron que la estimulación bilateral de los nervios del plexo pélvico produce una contracción vesical con presiones de 51 cc de agua y que la estimulación unilateral ocasiona solamente contracción vesical ipsilateral y presiones vesicales de $18 \mathrm{cc}$ de agua. No se sabe si la disfunción vesical es provocada por una sección de la inervación, por distensión nerviosa durante la tracción de la vejiga o por lesión térmica, pero por el tiempo de duración parece ser más bien una de las dos últimas causas en vez de la transección. De todas formas, la disección agresiva de la UUV, la coagulación excesiva o las suturas de anclaje ureteral si se realiza la variante de Zaont pueden ocasionar alteración de la inervación ${ }^{6,19,21}$. En esta serie, la disección ureteral fue mínima y limitada a la cara ventral del uréter y a la unión ureterovesical para evitar la lesión de los nervios perivesicales y la disfunción del vaciado. Para evitar la disfunción vesical, Leissner et al. ${ }^{19}$ recomiendan que los reimplantes ureterales extravesicales bilaterales se hagan en dos sesiones quirúrgicas separadas tres meses entre sí. Otros autores ${ }^{14,21}$ recomiendan no emplearla en niños con historia de disfunción vesical previa. A raíz de este estudio, hemos hecho algunas modificaciones técnicas en los reimplantes extravesicales bilaterales con el fin de minimizar el daño a las fibras nerviosas del plexo pélvico, que puede derivar en una disfunción vesical retentiva transitoria. En estos casos la disección del detrusor se hace de forma exclusiva con tijeras de Metzenbaum; la cauterización, si es necesaria, se realiza con las pinzas bipolares. Evitamos la disección en Y de la unión ureterovesical, limitando la disección del uréter distal hasta donde cruzan los vasos tributarios de la vesical inferior.

La administración de anticolinérgicos en el postoperatorio inmediato no parece ejercer ningún papel en la retención urinaria. Estos se utilizan sistemáticamente en todos los procedimientos quirúrgicos que requieren sonda vesical postoperatoria y cuando se retiran sonda y medicación todos los pacientes presentan hábito miccional normal.

Las náuseas y vómitos, que hasta hace poco carecian de importancia, les hemos dado valor porque alargan la estancia hospitalaria y el gasto. Cuando se compararon las complicaciones, días con sonda vesical y estancia hospitalaria de los procedimientos extravesicales con los intra se observaron diferencias estadísticamente significativas en el número de complicaciones inmediatas como dolor y hematuria, dias con sonda vesical y estancia hospitalaria que fueron mayores en los reimplantes tipo Cohen (Tabla 8).

Los factores que influyen en la estancia hospitalaria son: edad del niño, morbilidad asociada, procedimiento quirúrgico, tiempo operatorio $\mathrm{y}$ manejo postoperatorio ${ }^{7}$. Por todas estas razones el antirreflujo de Lich-Gregoire reúne los requisitos necesarios para ser un procedimiento de corta estancia hospitalaria.

Actualmente, el tratamiento endoscópico con el dextranómero del ácido hialurónico es una alternativa atractiva para el tratamiento quirúrgi- 
co del RVU. El procedimiento es ambulatorio y requiere una mínima analgesia postoperatoria. Sin embargo, la eficacia y morbilidad a largo plazo no se ha establecido todavía. La resolución del RVU a corto plazo varía del 70 al 89\% para reflujos grado I a $\mathrm{IV}^{23,24}$ en contraposición al reimplante vesicoureteral a cielo abierto que a largo plazo tiene un mayor porcentaje de curación (entre el 94 y 99\%) ${ }^{7-9,13,14}$.

\section{CONCLUSIONES}

1. La técnica de Lich-Gregoire es efectiva para la corrección del RVU uni y bilateral.

2. Los niños operados con los procedimientos extravesicales tienen un mejor postoperatorio inmediato que los operados con los intravesicales: menores molestias, dolor y hematuria.

3. La técnica de Lich-Gregoire puede ocasionar disfunción vesical transitoria en los procedimientos bilaterales (8,6\% en nuestra serie).

4. Es un procedimiento de corta estancia hospitalaria.

Este trabajo se realizó durante la estancia del primer autor en el Miami Children's Hospital con una beca de la Fundación para Investigación en Urología para estancia en un Hospital extranjero.

\section{REFERENCIAS}

1. Stein R, Thüroff JW. Correction of vesicoureteral reflux: where do we stand. Curr Opin Urol. 2004;14(4): 219-225.

2. O’Donnell B. Reflections on reflux. J Urol. 2004;172(4Pt2): 1635-1636.

3. Lich R II, Howerton LW, Davis LA. Recurrent urosepsis in children. J Urol. 1961;86:554-560.

4. Gregoire W. Le traitement chirurgical du reflux vesico-ureteral congenital. Acta Chir Belg. 1964;63:432-440.

5. Zaont MR, Maizel M, Sugar EC, Firlit CF. Detrusorrhaphy: extravesical ureteral advancement to correct vesicoureteral reflux in children. J Urol.1987;138(4 Pt 2):947-949.

6. Lipski BA, Mitchell ME, Burns MW. Voiding dysfunction after bilateral extravesical ureteral. Reimplantación. J Urol. 1998;159(3):1019-1021.

7. Marotte JB, Smith DP. Extravesical ureteral reimplantations for the correction of primary reflux can be done as outpatient procedures. J Urol. 2001;165(6Pt2):2228-2231.

8. Fung LCT, McLoire GA, Jain U, Khoury AE, Churchil BM. Voiding efficiency after ureteral reimplantation: A comparison of extravesical an intravesical techniques $J$ Urol. 1995;153(6): 1972-1975.

9. Burbige KA, Miller M, Connor JP. Extravesical ureteral reimplantation: results in 128 patients. J Urol 1996;155 (5):1721-1722.

10. David S, Kelly C, Poppas DP. Nerve sparing extravesical repair of bilateral vesicoureteral reflux: description of technique and evaluation of urinary retention. J Urol 2004;172 (4Pt2): 1617-1620.
11. Park JM, Retik AB. Surgery for vesicoureteral reflux. En Pediatric Urology, Gearhart JP, Rink RC, Mouriquand PDE eds. W. B. Saunders Company. Philadelphia. 2001;421429.

12. Elder JS. Guidelines for considerations for surgical repair of vesicoureteral reflux. Curr Opin Urol. 2000;10(6)579-585.

13. Barreiras D, Lapointe S, Reddy PP, Williot P, McLoire GA, Bagli D et al. Urinary retention after bilateral extravesical ureteral reimplantation: does dissection distal to the ureteral orifice have a role? J Urol. 1999;162(3Pt2):11971200 .

14. Minevich E, Aronoff D, Wacksman J, Sheldon CA. Voiding dysfunction after bilateral extravesical detrusorrhaphy. J Urol .1998; 160(3Pt2):1004-1006.

15. Hoenig DM, Diamond DA, Rabinowitz R, Caldamone AA. Contralateral reflux after unilateral ureteral reimplantation. J Urol. 1996;156(1):196-197.

16. Minevich E, Wacksman J, Lewis AG, Sheldon CA. Incidence of contralateral vesicoureteral reflux following unilateral extravesical detrusorrhaphy (ureteroneocystostomy). J. Urol. 1998;159(6):2126-2128.

17. Kumar R, Puri P. Newly diagnosed contralateral reflux after successful unilateral endoscopic correction: is it due to the pop-off mechanism. J Urol. 1997;158(3):1213-1215.

18. Diamond DA, Rabinowitz R, Hoenig DM, Caldamone AA. The mechanism of new onset contralateral reflux following unilateral ureteroneocystostomy. J Urol. 1996;156(2Pt2): 665-667.

19. Leissner J, Allhoff EP, Wolf W, Feja C, Höckel M, Black Pet al. The pelvic plexus and antirreflux surgery: topographical findings and clinical consequences. J Urol. 2001;165 (5): 1652-1655.

20. Yucel S, Baskin LS. Neuroanatomy of the ureterovesical junction: clinical implications. J Urol. 2003;170(3):945948.

21. Martínez-Portillo FJ, Seif C, Braun PM, Böhler G, Osmomov DK, Leissner Jet al. Risk of detrusor denervation in antirreflux surgery demonstrated in a neurophisiological animal model. J Urol. 2003;170(2):570-574.

22. Houle AM, McLoire GA, Heritz DM, McKenna PH, Churchil BM, Khoury AE. Extravesical nondismembered ureteroplasty with detrusorrhaphy: a renewed technique to correct vesicoureteral reflux in children. J Urol. 1992;148 (2):704-707.

23. Kirsch AJ, Perez-Brayfield, MR, Scherz, HC. Minimally invasive treatment of vesicoureteral reflux with endoscopic injection of dextranomer/hyaluronic acid copolymer: the Children's Hospitals of Atlanta experience. J Urol. 2003; 170(1):211-215.

24. Kirsch AJ, Perez-Brayfield M, Smith EA, Scherz, HC: The modified sting procedure to correct vesicoureteral reflux: improved results with submucosal implantation within the intramural ureter. J Urol. 2004;171(6):2413-2416.

Dr. M. García Mérida

E-mail: mgmerida@terra.es

(Trabajo recibido el 14 de febrero de 2006) 